\title{
Cause of death variation under the shared socioeconomic pathways
}

\author{
Samuel Sellers ${ }^{1}$
}

Received: 10 November 2019 / Accepted: 10 August 2020 /Published online: 23 August 2020

(C) Springer Nature B.V. 2020

\begin{abstract}
Climate change will create numerous risks for human health, including impacts associated with temperature extremes, diarrheal diseases, and undernutrition. Such risks, along with other socioeconomic and development trends, will affect cause-of-death patterns experienced in the coming decades. This study explores future mortality trends using the shared socioeconomic pathways (SSP) framework, a widely utilized tool for understanding socioeconomic development trends in a world with climate change. Existing projections for GDP, urbanization, and demographic trends based on SSP narratives are incorporated into an integrated assessment model, International Futures (IFs), in order to project mortality levels by cause of death for all countries from 2020 to 2100 . Under more optimistic SSPs, non-communicable diseases (NCDs) rise as a proportion of all deaths, particularly in low- and middle-income countries, while more pessimistic SSPs suggest a continued high burden of largely preventable communicable diseases. In highincome countries, significant continued burdens of NCDs are projected for the remainder of the century under all SSPs. Comparisons are also made to recent cause-of-death projections from the Institute for Health Metrics and Evaluation (IHME) to assess how the IFs and IHME models vary.
\end{abstract}

Keywords Mortality · Demography $\cdot$ Health $\cdot$ Traffic accident $\cdot$ International Futures

\section{Introduction}

In recent years, the literature linking global climate change and human health outcomes has rapidly developed. Climate change has a variety of adverse impacts on human health through

Author was affiliated with this institution when manuscript was composed, but is no longer affiliated.

Electronic supplementary material The online version of this article (https://doi.org/10.1007/s10584-02002824-0) contains supplementary material, which is available to authorized users.

Samuel Sellers

samsellers@gmail.com

1 Center for Health and the Global Environment, University of Washington, Seattle, WA, USA 
multiple and complex pathways, including temperature extremes, droughts, impacts of natural disasters, infectious diseases, and undernutrition (Watts et al. 2019). A subset of this literature has focused on the mortality impacts of specific climate-related mechanisms (Lutz et al. 2014b; Gasparrini et al. 2015; Hasegawa et al. 2016).

While there is uncertainty regarding the future health impacts of climate change, making projection challenging, in general, health harms are likely to be greater at higher levels of warming (Ebi et al. 2018). Although there are numerous and highly visible impacts of climate change in high-income countries, the health effects of climate change will be felt most in low- and middle-income countries that have less capacity to respond to climate-related health hazards (Keim 2008). Factors associated with socioeconomic development, including educational attainment, health system quality, and GDP, are associated with health outcomes, including causes of death (Marmot 2005). In particular, socioeconomic development patterns will affect population age structures which in turn significantly affect mortality causes, as infants and children experience different levels of risk for a variety of causes of death than middle-aged adults and elderly populations (KC and Lutz 2017). Thus, both the effects of climate change as well as socioeconomic development characteristics will impact the rates and causes of mortality around the world during this century.

Although various studies have explored mortality impacts attributable to specific climate-related risk factors, less research has explored the aggregate impacts of cause of death changes under different socioeconomic futures. This is largely because of the complexity of such efforts, which require incorporating a vast amount of information about health and socioeconomic risk factors and their associations with all causes of mortality, tasks that few research teams can muster. This study is able to circumvent such challenges by utilizing a health module within the International Futures (IFs) Integrated Assessment Model (IAM) from Hughes (2019), and assessing a series of socioeconomic development pathways to project cause-of-death outcomes.

Given growing global attention to the effects of climate change on mortality, it is important to understand how different development trajectories, with varying levels of adaptation and mitigation responses to climate change, are associated with specific mortality outcomes so that resources can be appropriately allocated to improve population health. This study aims to fill an important gap in the literature, providing regional projections of causes of death for 15 causes and cause groups covering virtually every country under five distinct socioeconomic pathways.

\section{Background}

Demographic forecasts have been used for decades in order to generate future population compositions by age, sex, country, and most recently, by level of education, allowing for refined analyses of future demographic trends (Lutz and KC 2011; Gerland et al. 2014). The accuracy of these forecasts has been improved over time through the inclusion of socioeconomic variables (Lutz et al. 2014a). For instance, regarding fertility, educational attainment (particularly for women), as well as access to family planning services, significantly affect desired and achieved fertility outcomes (Lutz and KC 2011; Bongaarts 2011). Additionally, socioeconomic inequalities are closely tied to disparities in health outcomes, including mortality (Marmot 2005). 
International migration is also affected by socioeconomic disparities, contributing to South-North migration flows (Castles 2013).

Recent research has sought to add further detail to demographic forecasts, in particular, by providing estimates of the mortality burdens associated with specific causes of death. Changes in age structures will occur alongside transitions in socioeconomic development and technology, which impact mortality rates and causes. Epidemiologists have found that in general, as development levels increase, causes of mortality shift from communicable to noncommunicable diseases (NCDs), a process referred to as the epidemiological transition (Orman 2005). This shift has a substantial impact on health systems in terms of what physical infrastructure, knowledge, and services are required in order to keep patients healthy.

While historical cause-of-death data are captured in local and national databases, collating these data across countries has been a considerable research challenge. In recent years, the Institute for Health Metrics and Evaluation (IHME) has made tremendous strides in doing this work through its Global Burden of Disease (GBD) study, most recently producing estimates for over 280 causes of death for every country from 1990 to 2017 (Roth et al. 2018). GBD has resulted in the harmonization of a great deal of knowledge concerning specific risk factors and mortality outcomes, including distal drivers, such as education level or income, as well as proximate drivers, such as smoking or occupational heavy metal exposures (Stanaway et al. 2018). In recent years, GBD has extended its work to include forecasting, using dozens of proximate and distal drivers, although this work has yet to incorporate the effects of climate change (Foreman et al. 2018).

Climate change presents substantial challenges to health forecasting, given its potentially unprecedented effects on a variety of distal and proximate drivers of health outcomes, including mortality (Watts et al. 2019). Climate change will affect the capacity of individuals to protect their own health, as higher levels of warming are likely to make adaptation to changing conditions more challenging, particularly for the poorest populations (Ebi et al. 2018). Most of the research on climate change and mortality relationships to date has centered on projecting impacts from a single cause or small group of related causes, such as mortality associated with heat (Gasparrini et al. 2015) or undernutrition (Hasegawa et al. 2016). Despite research on these and other climate change-mortality relationships, relatively little research has explored the potential aggregate impacts of climate change on multiple cause-of-death outcomes. A key exception is a 2014 study projecting impacts from six different causes of mortality: heat, coastal floods, diarrheal diseases, malaria, dengue, and undernutrition, finding hundreds of thousands of excess deaths per year worldwide from these causes by the 2030 decade, with impacts worsening by the 2050 decade (Hales et al. 2014).

However, health impacts associated with climate change will depend not only on the level of warming but also on how societies and their economies develop in the coming decades. These development patterns in part reflect public policy choices, including how governments and health systems prepare for climate change. These choices will affect the quantity and quality of care that can be provided in future decades, directly impacting population health outcomes (Sellers and Ebi 2018). While socioeconomic development pathways are not fully independent of the level of warming that will likely be experienced (for instance, a trajectory that reflects a low level of ambition for climate mitigation is very unlikely to coexist with a world with low levels of future warming), a range of socioeconomic development outcomes 
is possible at particular levels of warning. It is important to understand how the health outcomes of these trajectories are likely to vary in order to more fully understand health impacts in a changed climate.

This study provides cause-of-death projections that incorporate the effects of socioeconomic development and assumptions regarding adaptation and mitigation policy choices, though without modeling specific levels of emissions. These projections are designed to provide guidance for policymakers and scholars seeking to understand how climate change can impact all causes of mortality. In order to derive these projections, a series of socioeconomic pathways nested within an IAM are used. In addition, these projections are compared to projections recently developed by IHME's new forecasting model to illustrate the similarities and differences between IFs and IHME's forecasts. While there are many similarities between the models, the intention behind this comparison is not to suggest a particular model as being more or less accurate, but rather to illustrate the variation in projections generated to help policymakers and scholars understand how mortality outcomes may vary in future decades. A discussion of the scenarios used and the IFs and IHME models follows.

\section{Methods}

\subsection{The shared socioeconomic pathway framework}

In recent years, scholars have made advances in scenario development around potential futures in a world where the mitigation and adaptation challenges associated with climate change influence socioeconomic development. A major framework used to understand socioeconomic and political responses to climate change is the shared socioeconomic pathways (SSPs). The five SSPs are qualitative narratives that represent different socioeconomic futures, including different levels of climate adaptation and mitigation activities (O'Neill et al. 2017). Aspects of these narratives have also been quantitatively modeled, including urbanization (Jiang and O’Neill 2017), GDP (Dellink et al. 2017), income per capita (Crespo Cuaresma 2017), Human Development Index values (Crespo Cuaresma and Lutz 2015), and population (KC and Lutz 2017). The SSPs are the most up-to-date and widely used narratives for assessing how socioeconomic development trajectories will impact responses to climate change, replacing those presented by the Special Report on Emissions Scenarios (IPCC 2000). The SSPs can also be used to help understand potential cause-of-death trends in the coming decades, which is the primary objective of this paper.

By serving as a representation of sustainable development policies in a world with a changing climate, modeling using the SSPs provides a platform upon which to understand future mortality trends. However, the SSPs do not specify particular levels of emissions. In other research, SSPs are complemented by representative concentration pathways (RCPs), which model levels of radiative forcing (Van Vuuren et al. 2011). SSPs are designed to be used alone or in combination with the RCPs. For the analysis below, which is focused on socioeconomic responses to the challenges posed by climate change, RCPs are not used. However, previous literature notes that some SSPs and RCPs are incompatible with each other, suggesting that each SSP corresponds to a subset of potential radiative forcing levels (van 
Vuuren et al. 2014). In other words, some of the SSPs modeled below may not be achievable if emission levels are too high (or too low).

Briefly, SSP1 represents the most optimistic pathway, where challenges to adaptation and mitigation are low, resulting in global gains in socioeconomic development outcomes and health system adaptation capacity. SSP3 represents the most pessimistic pathway, where challenges to adaptation and mitigation are high, resulting in considerable difficulties in improving health services as a result of resource constraints and the impacts of climate-related disasters on economic growth. SSP2 represents an intermediate pathway between SSP1 and SSP3. Under SSP4, challenges to mitigation are low, but adaptation challenges are high, particularly in low- and middle-income countries, resulting in growing global socioeconomic inequality. SSP5 presents the opposite case - a world where adaptation to the effects of climate change is driven by fossil fuel use, resulting in less climate change mitigation, but continued economic growth and improvements in many socioeconomic indicators. Figure 1 displays the SSPs. Sellers and Ebi (2018) provide further detail on the differences between SSPs in terms of their assumptions and implications for health system responses.

Though the SSPs make no assumptions regarding the impact of radiative forcing, the assumptions inherent in these narratives regarding adaptation and mitigation are useful for thinking about how specific causes of mortality associated with climate

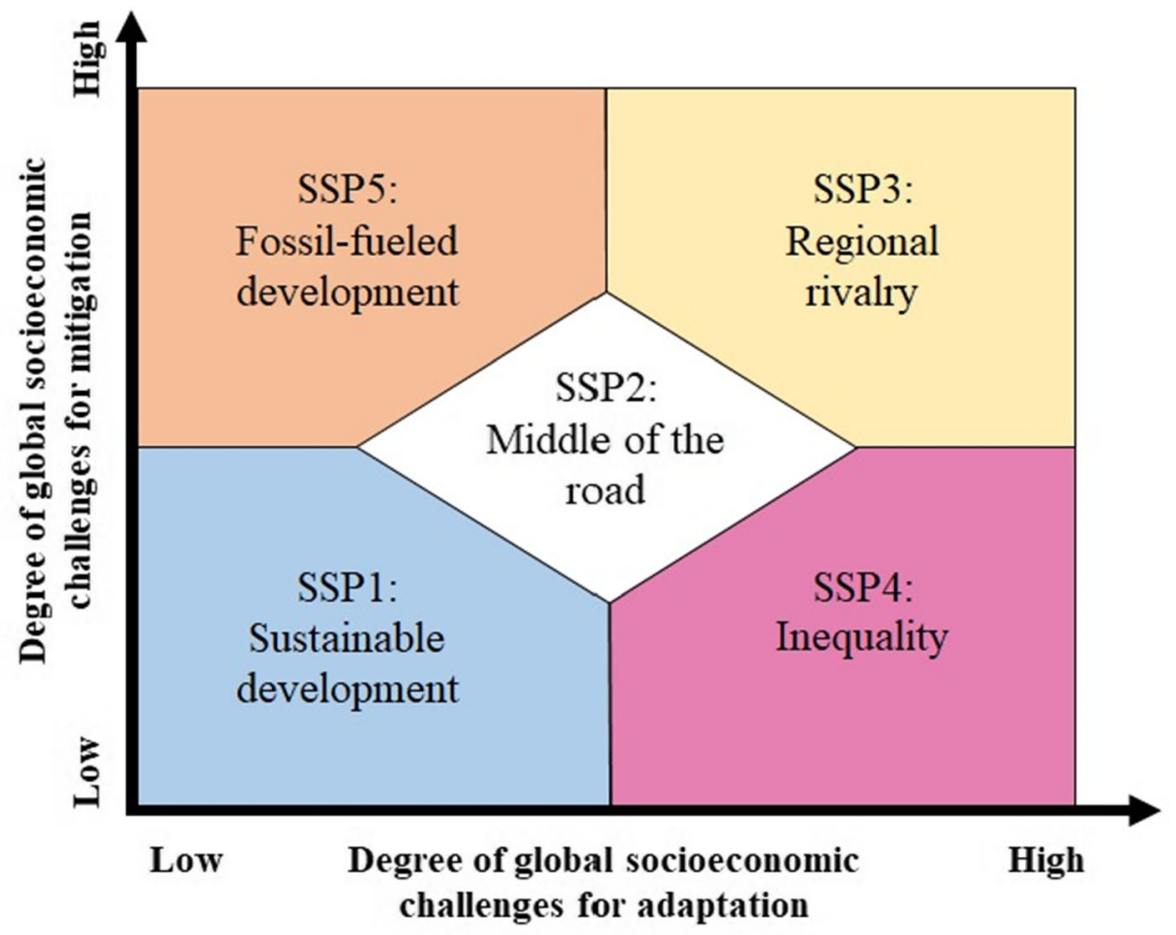

Fig. 1 Shared socioeconomic pathway framework 
impacts are likely to vary. For instance, changing rainfall patterns along with flooding and other natural disasters have the potential to reduce the quantity and quality of water available to households, particularly in settings where access to improved sources of water is low, which can in turn increase the frequency of diarrheal diseases (Howard et al. 2016). Similarly, poor adaptation planning and a lack of mitigation are projected to result in substantial crop losses in low- and middle-income countries that will increase the rate of poor health outcomes associated with undernutrition, particularly among children (Lloyd et al. 2011; Wheeler and von Braun 2013).

Additionally, there are health co-benefits associated with certain climate change mitigation strategies. For instance, indoor air pollution from dirty cooking fuels such as dung or wood is a major health hazard in many low-income countries, and this burden predominantly affects women and children (Jerneck and Olsson 2013). Burning these fuels not only produces harmful pollutants that can result in respiratory illnesses but also releases greenhouse gasses that exacerbate climate change (Anenberg et al. 2013). Initiatives to transition households to cleaner cooking technologies are thus important for mitigation, and are also likely to result in substantial reductions in mortality associated with respiratory illnesses. Additional health co-benefits are likely to accrue from efforts to reduce sources of outdoor air pollution, such as power plants and automobiles (Markandya et al. 2018).

The IFs model incorporates these linkages between proximate risk factors and specific cause categories (discussed further below) to illustrate some of the key relationships between sustainable development and mortality outcomes. However, some linkages addressed in the climate and health literature, and that are implicit in the SSPs, such as between governance capacity and the reduction of heat-related mortality (Lass et al. 2011; Hess and Ebi 2016), have not been incorporated into the IFs health module.

Age structures are also expected to change markedly between different SSPs, with SSP1 and SSP5 resulting in a steadily aging world population through lower fertility in low- and middle-income countries, but slightly higher fertility from current levels in many high-income countries. Under these pathways, children as a share of the world's population will decline, while elderly populations will grow, with the global population projected to peak at under nine billion around the middle of the century. By contrast, under SSP3 and SSP4, the global population will continue to grow substantially through relatively high fertility in low- and middle-income countries, resulting in large numbers of children vulnerable to premature death. Under SSP3, the global population does not peak by 2100 , at which time 12.6 billion people are expected to be alive (KC and Lutz 2017).

\subsection{The International Futures Integrated Assessment Model}

To generate cause-of-death projections, this study uses the IFs IAM, which projects a variety of socioeconomic, environmental, and development outcomes in future world states. IFs is a demonstrated tool in the health literature and has been used in prior research to project future health outcomes using different socioeconomic assumptions (Hughes et al. 2011; Kuhn et al. 2016). Additionally, it is viewed as one of the strongest tools available for modeling Sustainable Development Goals outcomes (Allen et al. 2016). 
IFs contains a health module enabling users to project mortality by cause associated with different socioeconomic development assumptions, including those modeled using the SSPs. As an IAM, IFs contains a series of modules that are interconnected and have feedbacks, allowing for health outcomes, for instance, to be impacted by calculations in other modules, including those related to governance, economic development, and infrastructure. When the SSPs are applied to IFs, the effects of differences in adaptation and mitigation capacity on specific causes of mortality are illustrated mostly through changes in the levels of proximate risk factors (described further below).

The following description of the IFs health module summarizes more extensive discussions found in other sources that interested readers should consult (Hughes et al. 2014; Kuhn et al. 2016; Hughes 2019). IFs combines distal and proximate drivers of mortality outcomes in order to assign causes of death to particular age-sex-country-year categories. The IFs health module builds on research from Mathers and Loncar $(2005 ; 2006)$, who estimated mortality for ten cause-of-death categories between 2003 and 2030 using distal drivers of health outcomes, including country-level data for GDP per capita (PPP) and years of education (among individuals age 25 and above), as well as a variable for time (as a proxy for technological innovation), and for some cause categories, a measure of smoking impact. ${ }^{1}$ Using these distal drivers, separate regressions were run for each cause category by sex and age group $(0-4,5-14,15-29,30-44,45-59,60-69$, and 70 and older) using global data from 1950 to 2002 in order to generate mortality projections forward to 2030 . In addition to coefficients generated using a panel of all countries, a separate set of models using the procedures above was run only using historic data from low-income countries, with separate coefficients reported.

IFs adopts this framework to generate its own cause-of-death projections for 15 causes and cause categories, but makes several key modifications in order to derive its projections (Table 1).

First, IFs generates projections for age groups in line with standard demographic methods, typically for 5-year intervals except for age $0-1,1-4$, and 100 and older categories. IFs adopts the coefficients generated using the distal drivers above and applies them to all of the 5-year age groups within the broader age groups used by Mathers and Loncar (2006). Specific adjustments are made in IFs to account for infant mortality rates (which tend to be higher than mortality in the 1-4 age group), as well as to ensure that non-communicable cause group death rates increase monotonically for age groups starting at age 45. Additionally, the coefficients generated by Mathers and Loncar for low-income countries are used in IFs for countries with a GDP below $\$ 3000$ (PPP), and the full model coefficients for countries with GDP above $\$ 15,000$, with an averaging procedure applied using both sets of coefficients for countries in between these levels.

Second, IFs adjusts projections for select cause categories using a series of proximate risk factors. Assessments of the population attributable fraction of specific proximate risk factors are used to modify projections of mortality for specific cause categories from those generated by the distal driver approach above based on equations derived from other mortality analyses.

\footnotetext{
${ }^{1}$ Three related but distinct terms are used here to describe causes of death, with varying levels of specificity. "Cause" refers to a specific cause of death, which, depending on the condition, may include deaths associated with more than one ICD-10 code. "Cause category" refers to multiple causes that are typically associated with a similar set of risk factors. For instance, in IFs, cardiovascular diseases are modeled as a single-cause category, which encompasses deaths from ischemic heart disease and strokes, among other causes. "Cause group" refers to three specific groups of causes outlined by the GBD study, under which all cause categories and causes fall: communicable (Group 1), non-communicable (Group 2), and injuries (Group 3).
} 
Table 1 List of IFs causes of death by cause group

\begin{tabular}{lll}
\hline $\begin{array}{l}\text { Group I causes (communicable } \\
\text { diseases) }\end{array}$ & $\begin{array}{l}\text { Group II causes (non-communicable } \\
\text { diseases) }\end{array}$ & Group III causes (injuries) \\
\hline $\begin{array}{l}\text { Diarrheal diseases } \\
\text { Malaria }\end{array}$ & $\begin{array}{l}\text { Malignant neoplasms } \\
\text { Cardiovascular diseases }\end{array}$ & $\begin{array}{c}\text { Road traffic accidents } \\
\text { Other unintentional } \\
\text { injuries }\end{array}$ \\
Respiratory infections & Digestive diseases & Intentional injuries \\
HIV/AIDS & Chronic respiratory diseases \\
Other group I causes & Diabetes & \\
& Mental health & \\
& Other group II causes & \\
\hline
\end{tabular}

Proximate risk factors used in the IFs model include calories per capita (for communicable diseases associated with undernutrition), governance capacity and social stability (for childhood communicable diseases), water and sanitation access (for diarrheal diseases), indoor solid fuel use (for respiratory diseases), outdoor air pollution (for respiratory diseases, cardiovascular diseases, and respiratory infections), and body mass index (BMI; for cardiovascular diseases and diabetes). These proximate risk factors help the model reflect how differences in adaptation and mitigation approaches, as expressed through the ability of governments to reduce pollution, improve nutritional outcomes, develop sanitation infrastructure, and govern effectively, among other steps, impact specific mortality cause categories.

Third, two causes of death (AIDS and road traffic deaths) are calculated with specialized submodels using a different set of methods from the distal/proximate driver approach detailed above. However, upon assessing the projections generated using the road traffic submodel, which were substantially higher than projected from other sources, the author opted to model these deaths using a different set of modeling approaches based on recent World Health Organization (WHO) analyses (Supplementary Appendix).

Fourth, IFs contains the capability to specify certain inputs exogenously using projections derived from outside sources. It is through specifying exogenous inputs that the SSPs can be represented in the IFs model. To do this, default values in IFs are replaced with exogenous projections for key variables, including total population size, births, deaths, fertility and migration rates, and educational attainment for individuals above age 15 and age 25 (KC and Lutz 2017), as well as urbanization rates (Jiang and O’Neill 2017), and GDP per capita (PPP) (Dellink et al. 2017). ${ }^{2}$ The values from these projections influence parameters endogenously calculated within the IFs model, and affect both the distal and proximate determinants of various mortality outcomes.

The results below summarize global mortality outcomes under the five SSPs as projected in IFs. Model results are presented at the global level and by region, with countries organized into

\footnotetext{
${ }^{2}$ When the original SSP population projections were made by KC and Lutz (2017), United Nations 2010 population data were used as a base, with projections carried forward to 2100 . As new data have come in, IFs uses United Nations 2015 data as a base (United Nations 2019). The use of newer data allows for more accurate projections, particularly as fertility decline in many low-income countries has proceeded more slowly than previously anticipated. In IFs, the year-on-year percentage changes in demographic variables generated by $\mathrm{KC}$ and Lutz in their original projections by age-sex-country-year have been carried forward and applied to the 2015 base data. As a result, the values calculated by IFs for demographic variables, including for number of deaths, do not exactly match those originally calculated by $\mathrm{KC}$ and Lutz, but are generally quite similar. Differences are also found between the GDP and urbanization projections made in IFs, which reflect more recent base data from the World Bank (2018), and the United Nations (2018) respectively, and the raw values in the original publications.
} 
seven regions, six based on geography, and a single region representing all high-income countries as of 2019 (Table S1). These regional groupings were selected to match those used by Hughes et al. (2011).

\subsection{IHME forecasts}

In 2018, IHME published cause-of-death forecasts through the year 2040; a brief description of the model is provided here, further details about their modeling procedures can be found in the referenced publication (Foreman et al. 2018). The IHME model has three main components. The first component incorporates the relationship between the relative risk of 65 risk factors and specific cause-of-death outcomes. The second component consists of a distal drivers approach similar to that outlined above under IFs, using a series of socioeconomic covariates, with adjustments based on established relationships between certain covariates with specific causes of death. Finally, the model includes an autoregressive moving average component to address residual variation. The author downloaded IHME's point estimates on individual causes of death at year 2040 and compared them to results found in IFs using the SSPs for 2040. Point estimates were used rather than the full confidence intervals to facilitate comparability, as IFs estimates are not probabilistic; interested readers should consult the Foreman et al. (2018) publication for IHME's full probabilistic findings. Because IHME's forecasts provide estimates for 250 causes of death, each listed cause was grouped into one of the 15 categories modeled by IFs by matching the ICD-10 codes provided by IHME for each cause to the cause categories used by IFs to facilitate comparability across models.

In addition to a reference forecast, IHME generated two scenario forecasts by making different assumptions of the rates of change of distal and proximate predictors of mortality outcomes used in their model. A better health forecast was generated using the 85 th percentile of the rate of change from 1990 to 2016 of predictors used in their model, while a worse health forecast used the 15th percentile of these rates. These scenarios are somewhat analogous to the SSPs, insofar as the SSPs make more or less optimistic assumptions regarding the trajectories of distal and proximate drivers of mortality outcomes. However, unlike the SSPs, the IHME better/worse health scenarios were not developed using any assumptions or narratives regarding the effects of adaptation or mitigation to the effects of climate change.

\section{Results}

Global mortality will increase in the coming decades, with annual deaths rising under SSP3 from 67.5 million in 2020 to 109.6 million in 2050 , and 146.5 million by 2100 , figures that are in line with earlier analyses (KC and Lutz 2017). By contrast, overall mortality is expected to rise much more slowly under SSP1 and SSP5, largely because of slower overall population growth as well as longer life expectancies under these pathways. These scenarios are largely indistinguishable in terms of overall mortality trajectories, with mortality expected to rise from $61.2-61.3$ million in 2020 to $77.3-77.5$ million in 2050 , and $105.0-105.1$ million by 2100 (Fig. 2). These mortality trajectories are closely connected to changes in average life expectancy. Under SSP1, global average life expectancy is projected to reach 82.0 years by 2050 and 90.1 years by 2100. A far bleaker future is envisioned under SSP3, where average life expectancy is only projected to reach 73.5 years by 2050 and 75.2 years by 2100 . 


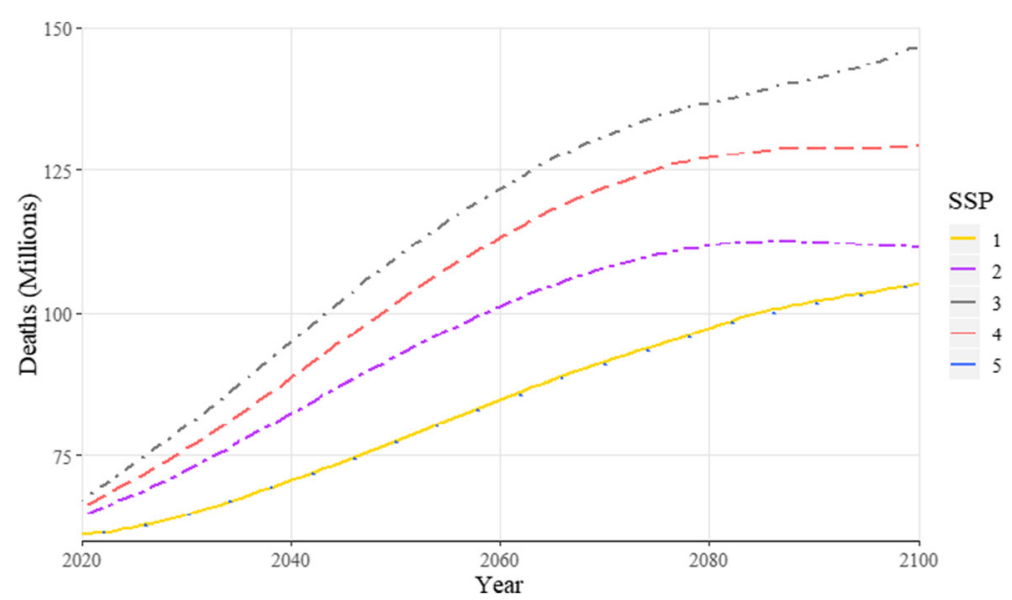

Fig. 2 Global mortality trajectories under each SSP using IFs, 2020-2100. (Note: SSP5 has a very similar trajectory to SSP1, so it is displayed as lightly dashed)

Globally, mortality is expected to remain heavily concentrated in non-communicable causes of death (Figs. S1-S3). However, under SSP3 and SSP4, communicable diseases and injuries will comprise a greater fraction of mortality in low- and middle-income countries than under more optimistic pathways. While NCDs will continue to constitute the vast majority of mortality worldwide, patterns will vary by region. Sub-Saharan Africa will continue to have a disproportionately high burden of communicable diseases into the future, particularly under more pessimistic SSPs. A similar pattern, albeit less pronounced, will occur in South Asia. By contrast, in high-income countries, as well as most other world regions, NCDs as a fraction of all deaths will remain high throughout the century, even under the most pessimistic SSPs (Fig. 3). Communicable disease deaths are expected to fall in absolute terms under all SSPs by 2100 , ranging from a total of 4.5 million deaths globally under SSP2 to 13.3 million deaths under SSP4. Injury deaths are projected to increase, even under the most optimistic SSPs, ranging from 10.1 million deaths under SSP1 to 18.1 million deaths under SSP3, largely a reflection of growing automobile use.

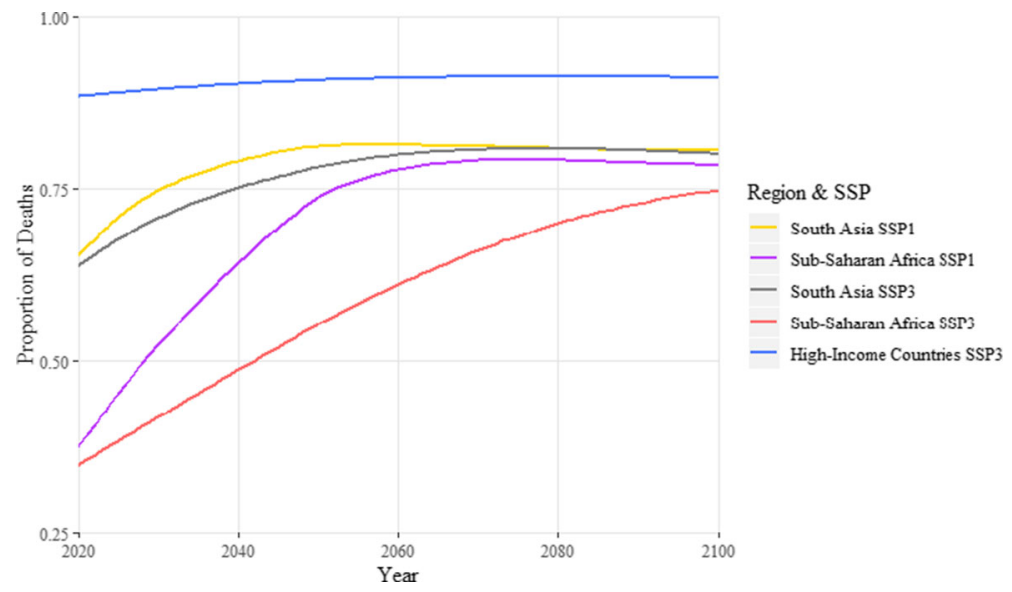

Fig. 3 Trends in share of NCD deaths from selected world regions/SSPs, 2020-2100. (Note: To facilitate readability, SSP1/high-income countries is omitted from this graph due to strong overlap with SSP3) 


\subsection{IFs cause-of-death results}

The following discussion of mortality largely centers on five of the 15 causes modeled in IFs: cardiovascular diseases, diarrheal diseases, chronic respiratory diseases, cancer, and road traffic accidents. These causes are highlighted because of their high or growing incidence (in the cases of cardiovascular diseases and cancer), their linkages with environmental conditions (in the cases of diarrheal and chronic respiratory diseases), or their linkages with socioeconomic development (in the case of road traffic accidents). In general, they do not represent the five largest causes of mortality within a particular region or year, but rather were selected to illustrate key trends. Fraction of overall mortality rather than absolute number of deaths is used in order to more easily compare the relative burdens of different causes across pathways and models. The years 2030, 2050, and 2080 are used as markers to illustrate how burdens will change over time. Subsequent to this discussion, IFs data are compared to IHME projections, which are for the year 2040.

Cardiovascular diseases Examining mortality by individual cause category, world region, and SSP, in 2030, 2050, and 2080, these projections illustrate that cardiovascular diseases will continue to be the single leading cause category of death worldwide (Figs. S4-S6). Cardiovascular diseases will constitute over $50 \%$ of all deaths in Europe \& Central Asia and roughly $40-42 \%$ in the Middle East \& North Africa under all SSPs by 2030. By contrast, cardiovascular diseases will constitute between 13.8 (SSP4) and 21.5\% (SSP5) of mortality in 2030 in sub-Saharan Africa, reflecting the continuing high burden of communicable diseases in the region. In future decades, cardiovascular disease burdens will decrease in most world regions as other NCDs become increasingly large causes of mortality. The notable exception is in subSaharan Africa, where, due to longer life expectancies and a faster reduction of communicable diseases in more optimistic SSPs, cardiovascular disease mortality is expected to rise as a share of all mortality by 2050 , before falling again by 2080 . Elevated cardiovascular mortality is in line with endogenous projections suggesting rising obesity levels, particularly under SSPs that assume faster development trajectories. For instance, in the Middle East \& North Africa region, roughly $32.3 \%$ of the population is projected to be obese by 2030 under SSP1, compared to $31.4 \%$ under SSP3. By contrast, $9.0 \%$ and $8.3 \%$ of the population in subSaharan Africa are projected to be obese under SSP1 and SSP3, respectively, as of 2030.

Diarrheal diseases Mortality from diarrheal diseases is heavily concentrated in South Asia and sub-Saharan Africa, and will represent up to $6.5 \%$ of all mortality in the latter region in 2030 , although deaths from diarrheal diseases as a share of all mortality are expected to fall towards the end of the century (Fig. 4). However, the diarrheal model in IFs is sensitive to age structure as well as to development and climate adaptation measures such as improved access to water and sanitation services. Thus, the model accounts for the fact that diarrheal diseases disproportionately affect the very young and very old, as well as the fact that infrastructure improvements can reduce the incidence of diarrheal diseases. In IFs, the more optimistic SSPs, SSP1, and SSP5, are actually projected to have a greater share of mortality attributable to diarrheal diseases than under SSP2 or SSP3 in South Asia and sub-Saharan Africa by 2080 in part because the age structure under the former pathways will be older than under the latter. In 2080, diarrheal disease mortality ranges from 2.6 of all deaths (SSP3) to 6.0\% (SSP4) in South Asia and from 1.7 (SSP2) to $3.8 \%$ (SSP4) in sub-Saharan Africa. 


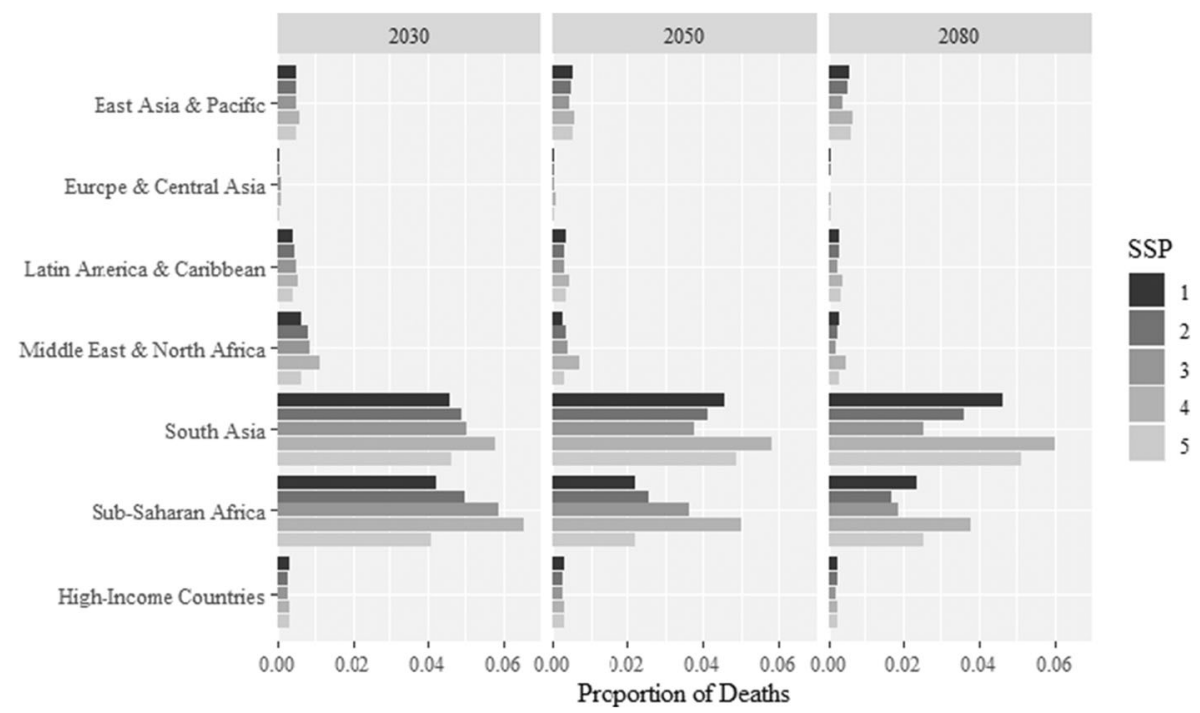

Fig. 4 Trends in diarrheal disease across world regions/SSPs, 2030, 2050, and 2080

While IFs projects considerable convergence across SSPs in water and sanitation indicators towards the end of the century, there are important differences between the trajectories in water and sanitation access indicators in the near term that affect diarrheal mortality across SSPs. For instance, in South Asia, IFs estimates that access to piped water will climb rapidly under SSP1, reaching $80.6 \%$ of the population by 2030 , but only $76.7 \%$ under SSP3. In sub-Saharan Africa, $63.1 \%$ of the population will have such access by 2030 compared to $57.5 \%$ under SSP3. In both regions, similar disparities are projected in access to improved sanitation services, with convergence between SSPs projected earlier in South Asia than in sub-Saharan Africa.

Chronic respiratory diseases Chronic respiratory mortality is heavily concentrated in South Asia and East Asia \& Pacific, with over $10 \%$ of mortality in both regions attributable to chronic respiratory diseases under all SSPs by 2030 . These conditions are significantly affected by indoor and outdoor air pollution concentrations. More optimistic development trajectories are likely to reduce deaths associated with indoor air pollution in the near term. For instance, IFs indicates that $19.8 \%$ of individuals in South Asia will reside in households that use wood, dung, or other solid fuels for cooking as of 2030 under SSP1, compared to 30.5\% under SSP3. East Asia will likely be further along in its transition to cleaner cooking technologies, with $5.8 \%$ and $11.0 \%$ of individuals expected to use solid fuels for cooking under SSP1 and SSP3, respectively, by 2030. However, even under more optimistic trajectories, outdoor air pollution concentrations are projected to be at high levels for some time, adversely affecting chronic respiratory mortality burdens. In South Asia, urban PM2.5 concentrations are projected to reach $43.9 \mu \mathrm{g} / \mathrm{m}^{3}$ by 2030 under SSP1 compared to $48.3 \mu \mathrm{g} /$ $\mathrm{m}^{3}$ under SSP3. In East Asia, comparative levels are lower, but still high at $25.1 \mu \mathrm{g} / \mathrm{m}^{3}$ under SSP1 $26.3 \mu \mathrm{g} / \mathrm{m}^{3}$ in SSP3. ${ }^{3}$ In future decades, despite increased implementation of pollution controls, GDP growth and aging populations are likely to result in an increased burden associated with chronic respiratory diseases, particularly in these two regions, and particularly under SSP1 and SSP5 which forecast higher rates of economic growth than other SSPs (Figs. S7-S9).

\footnotetext{
$\overline{{ }^{3} \text { For reference, the current PM } 2.5}$ limit for primary emissions in the USA is $12 \mu \mathrm{g} / \mathrm{m}^{3}$ (EPA 2013).
} 
Cancers Cancer deaths as a proportion of all mortality will remain relatively steady throughout most world regions, with the exception of sub-Saharan Africa, where such deaths are projected to rise from 9.4 to $13.1 \%$ of deaths in 2030 to between 17.1 and $28.8 \%$ by 2080 , with SSP4 experiencing the lowest burden and SSP5 the highest during both of these years (Figs. S10$\mathrm{S} 12)$. These trends are largely in line with the decline in mortality associated with communicable diseases. Slower growth will occur in South Asia, where as many as $15.3 \%$ of deaths may be attributable to cancer by 2080 under SSP5. In high-income countries, cancer-related mortality is likely to slowly decline during the century, but will still make up at least $20 \%$ of all mortality by 2080 .

Road traffic accidents Road traffic accident deaths are responsible for a substantial proportion of fatalities from injuries, representing a significant public health problem worldwide (Figs. S13-S15). Unsurprisingly, accident risks are projected to be greater in countries with poorer safety infrastructure and traffic law enforcement. In 2030, traffic accidents are likely to represent slightly less than $1 \%$ of all mortality in high-income countries, while accounting for as much as $4.4 \%$ of all mortality in the Middle East and North Africa (under SSP1). Projections are sensitive to GDP and growth in vehicles per capita, which are expected to be higher under optimistic SSPs, resulting in higher proportions of traffic fatalities in 2050 under SSP1 and SSP5, though this trend reverses itself by 2080. Readers should exercise caution when interpreting traffic fatality projections after 2050 due to the modeling methods used as well as potentially transformative technological and behavioral developments that may reduce traffic mortality (see Supplementary Appendix for additional details).

\subsection{Comparing IFs and IHME projections}

The following discussion compares findings from IFs with those from the recent IHME projections. Predicted cardiovascular mortality is relatively similar across both models, particularly for central projections. For instance, in the region where cardiovascular disease mortality is most common, Europe \& Central Asia, SSP2 projects roughly 50.3\% of deaths will be attributable to cardiovascular disease in 2040, which is identical to the IHME reference projection point estimate (Fig. S16). When the more pessimistic/optimistic scenarios are explored, the gap between the IFs and IHME projections grows. In the same region, under SSP3, $51.1 \%$ of deaths are projected due to cardiovascular disease versus $53.6 \%$ of deaths under the IHME worse health projection. Broadly similar patterns between the models are also found for cancer mortality (Fig. S17).

More substantial differences between the models exist for the three other causes of death highlighted. South Asia and Sub-Saharan Africa are notable in both models for having elevated shares of mortality for diarrheal diseases, even under more optimistic health scenarios (Fig. S18). However, the mortality fractions associated with diarrheal diseases vary between the models, with IHME being more pessimistic. For instance, in South Asia, IHME's reference projection estimates $4.7 \%$ of deaths would be attributable to diarrheal diseases in 2040, while the worse health projection estimates $7.2 \%$ of deaths attributable to diarrheal diseases in the same year. These estimates equate to roughly 792,000 and 1.59 million deaths, respectively. By contrast, IFs estimates $4.4 \%$ of deaths (roughly 765,000) will be attributable to diarrheal diseases under SSP2 in 2040 in South Asia, whereas under SSP4 (the most pessimistic SSP for diarrheal diseases), roughly $5.7 \%$ (1.03 million deaths) will be attributable to diarrheal 
diseases, a difference of roughly 450,000 deaths from IHME's model. A disparity between IFs and IHME findings also exists between the two models in sub-Saharan Africa, where IHME's reference and worse health projections are much more closely aligned, though it is less stark than in South Asia. These disparities suggest that the more pessimistic scenarios in the two models may be making differing assumptions for water and sanitation access and childhood undernutrition in many low- and middle-income countries, given its proximate effects on diarrheal disease mortality.

Concerning chronic respiratory diseases, these projections show higher proportions and numbers of deaths in the East Asia \& Pacific and South Asia regions than IHME (Fig. 5). These differences are likely related to differing assumptions about the relationship between GDP growth and air pollution, as well as the effect of air pollutant concentrations on mortality outcomes. In South Asia, for instance, IHME estimates air pollution will be responsible for

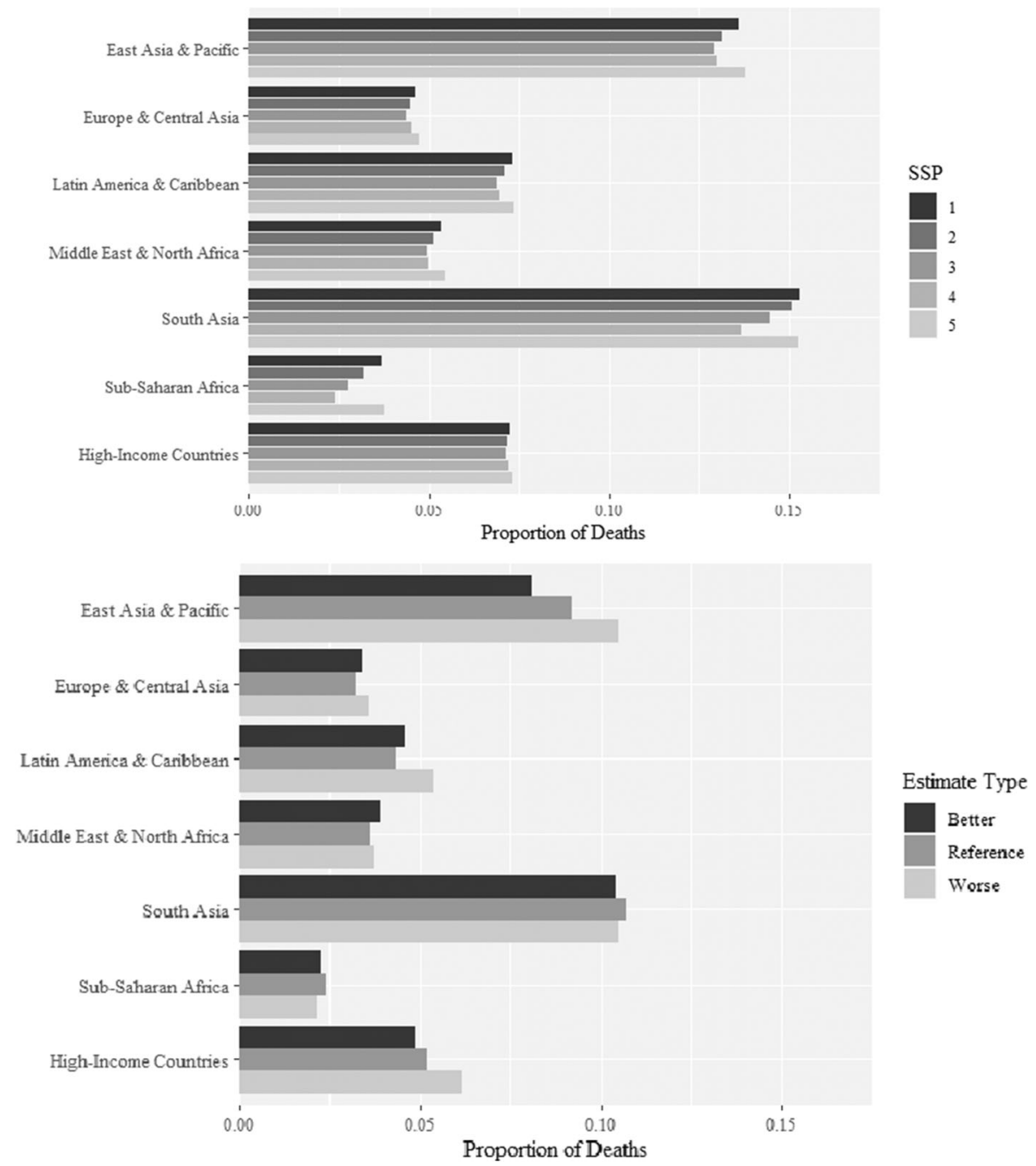

Fig. 5 Comparison of IFs (top) and IHME (bottom) findings on share of deaths from chronic respiratory disease by world region/SSP, 2040 
between 10.4 and $10.7 \%$ of deaths in 2040, while IFs places the total at $13.7-15.3 \%$ of deaths, a sizable gap. Ongoing efforts to improve environmental health estimates in the IHME's modeling may result in convergence between the two models over time (Shaffer et al. 2019).

IFs and IHME also exhibit key differences with regard to traffic mortality projections (Fig. S19). Despite adjusting IFs traffic projections to account for more recent WHO data and methods, a gap remains between IFs-generated projections and those from IHME, with IFs projections generally being higher. This gap is especially pronounced in sub-Saharan Africa, where up to $5.6 \%$ of deaths will be caused by traffic accidents in this region in 2040, under the modified traffic deaths procedure adopted in IFs, approximately twice the share that IHME projects. There is an important commonality between both models, namely that traffic fatalities as a share of mortality tend to be higher in sub-Saharan Africa under more optimistic pathways, a reflection of the high burdens of communicable diseases in the region that are unlikely to be reduced in more pessimistic pathways, lowering the proportion of deaths from traffic accidents which tend to disproportionately fall among workingage adults as opposed to children.

\subsection{Mortality by age group}

This final discussion concerns mortality by age group in the IFs model, with a focus on child mortality. Young children are especially vulnerable to communicable diseases, though this is particularly true in settings with limited health care capacity. However, the vulnerability of children to communicable diseases will vary considerably throughout the remainder of the century depending on socioeconomic development patterns, with changes most pronounced in the least developed regions of the world, South Asia and sub-Saharan Africa. In 2030, over seven in ten deaths of children age 0-4 in South Asia and sub-Saharan Africa are projected to be from communicable diseases, under all SSPs, and this remains true under SSP3 and SSP4 at 2050 (Fig. S20). By 2080, there is greater divergence between the SSPs, where fewer than $50 \%$ of deaths in children age $0-4$ in sub-Saharan Africa will result from communicable causes under SSP5, while more than $75 \%$ of deaths in that region are projected to be from communicable causes under SSP4. In high-income countries, over $40 \%$ of deaths in children age $0-4$ under all SSPs will be attributable to communicable causes in 2030. While this falls slowly but steadily over the century, burdens will still be substantial (over one in three deaths) under SSP3 and SSP4 by 2080. Substantial communicable disease burden among young children (over $50 \%$ of deaths in at least one SSP) will also be found in 2080 in East Asia \& Pacific and Europe \& Central Asia. While Fig. S20 displays share of total mortality, it is important to remember that the absolute numbers of deaths will vary significantly across SSPs because of changes in birth rates and infant mortality risks associated with distal and proximate drivers. For instance, in South Asia, 1.32 million children age 0-4 are projected to die (of any cause) under SSP3 in 2050, whereas under SSP5, this number falls to 162,000, a profound example of the divergent fertility and mortality outcomes exhibited by the SSPs.

\section{Discussion}

Mortality outcomes in the remainder of the twenty-first century may take a wide range of forms depending on the trajectories of adaptation and mitigation responses to the effects of climate change and the relationship between these trends with health care delivery and disease risk factors. Understanding mortality burdens in future decades can facilitate the efficient 
allocation of health care resources to treat the greatest population health risks. The substantial variation in projections between SSPs, particularly for communicable diseases, suggests that strong public health efforts to reduce exposure to risk factors and improve health care delivery in low- and middle-income countries can change mortality trajectories, in particular, by reducing the burden of communicable diseases on young children. This is especially necessary in sub-Saharan Africa and South Asia, where communicable disease burdens are substantial under more pessimistic SSPs, even towards the end of the century. Moreover, those regions are likely to experience growing burdens of NCDs as well, placing dual strains on health systems (Bygbjerg 2012). Additionally, the substantial health impacts attributable to pollution in these regions, most clearly seen in the relationship between ambient air pollution and chronic respiratory disease mortality, should motivate a stronger focus from policymakers on reducing pollution loads (Landrigan et al. 2018). Given the growing burdens on health care systems from NCDs in particular, care delivery models will need to shift further towards prevention through stronger efforts to ameliorate the social and environmental determinants of adverse health outcomes.

Demographic changes, in particular, aging populations, will likely affect continued vulnerability to communicable diseases. As noted above, these projections suggest elevated rates of death from diarrheal diseases under SSP1 and SSP5 in South Asia and sub-Saharan Africa in 2080, reflecting the heightened vulnerability of elderly populations to these diseases that has been documented in the latter half of the twentieth century. New treatments and public health measures will be needed in order to attenuate this relationship between population aging and diarrheal disease mortality, which very well may be developed by the latter half of the twentyfirst century, further reducing the burden of communicable diseases in the population. However, despite this challenge, SSP1 and SSP5 present a much healthier future for humanity overall, with longer life expectancies and far fewer children perishing from communicable diseases than under more pessimistic trajectories, allowing the vast majority of children in all parts of the world to develop into healthy adults.

The results presented also pose challenges for high-income countries. These countries have largely completed the epidemiological transition and are projected to have relatively low burdens of communicable diseases and injuries for the remainder of the century, even under more pessimistic SSPs. This reflects both advancements in health service delivery as well as the greater resources at the disposal of high-income countries to adapt to the health effects of climate change. Even as populations age, high levels of development will insulate countries from significant growth in communicable disease burdens.

Other regions of the world (East Asia \& Pacific, Europe \& Central Asia, Middle East \& North Africa, and Latin America \& Caribbean) are likely to experience intermediate patterns to those described above. The economic development that has occurred in these regions in the past several decades has resulted in improved public health outcomes such as better access to water and sanitation, reductions in indoor air pollution, and stronger enforcement of traffic safety laws. As is true in other regions of the world, slower and less sustainable forms of economic development in the twenty-first century are likely to result in worse health outcomes and greater burdens of disease from communicable causes.

Comparing the results of IFs and IHME projections in the year 2040 for share of overall mortality by cause found more similarities than differences across models. In particular, the trends outlined above regarding high NCD burdens in high-income countries and continued communicable disease burdens in low- and middle-income countries were common across both models. However, current IHME results are constrained by the lack of specific 
assumptions made regarding adaptation, mitigation, as well as overall levels of warming, all of which will influence human health outcomes associated with climate change.

The IFs projections presented here have important limitations. Most significantly, these projections center on changes in economic development, and do not directly measure the effects of climate change on mortality. Changes in climate conditions may generate feedback loops, for instance, increasing death rates for causes related to extreme heat or undernutrition, which in turn may slow economic development and worsen future health outcomes. Incorporating relationships between radiative forcing levels and multiple mortality causes will be a key step forward in facilitating health system adaptation.

Generating mortality projections in a world with climate change will continue to be a challenging exercise for the foreseeable future due to gaps in data availability as well as the general unpredictability of global change. On the one hand, the development of new cures or treatments for leading causes of death such as AIDS may result in longer life expectancies than currently projected and reduced (or eliminated) mortality burdens from specific causes. Moreover, a reduction in exposure to major risk factors, such as air pollution or contaminated water supplies, may allow low- and middle-income countries to proceed through the epidemiological transition more rapidly than projected. On the other hand, stronger-than-anticipated impacts of climate change on mortality, particularly in vulnerable regions, may result in shorter life expectancies and slower improvements in health outcomes. Additionally, the COVID-19 pandemic has highlighted the fragility of many of national health systems, which is an ominous sign for their ability to deal with future challenges associated with sudden-onset climate events. Given continued difficulty in mustering global action to mitigate climate change, as well as the lack of financial and human capital in low- and middle-income countries for adaptation activities, SSP3 appears to be an increasingly probable trajectory for much of the world, an extremely troubling development for population health outcomes.

As socioeconomic and health data become more widely available and more accurate, continued revision of these projections and model adjustment based on the most recent trends is essential. Moreover, as the effects of climate change become clearer, iterative scenario development, including for the SSPs, will be necessary in order to more accurately reflect the range of possible health outcomes in future decades. An advantage of the SSP framework is that it is amenable to iteration; as higher-quality data on mortality are collected for more countries and as our understanding of relationships between specific mortality causes and key socioeconomic or biophysical predictors is further understood, future mortality projections using the SSPs can and should be improved upon.

Acknowledgments Many thanks to Kristie L. Ebi and Jeremy Hess who provided guidance on framing this manuscript. Kanishka Narayan and Barry Hughes provided helpful technical assistance on using the IFs model.

\section{References}

Allen C, Metternicht G, Wiedmann T (2016) National pathways to the sustainable development goals (SDGs): a comparative review of scenario modelling tools. Environ Sci Pol 66:199-207. https://doi.org/10.1016/j. envsci.2016.09.008

Anenberg SC, Balakrishnan K, Jetter J et al (2013) Cleaner cooking solutions to achieve health, climate, and economic cobenefits. Environ Sci Technol 47:3944-3952

Bongaarts J (2011) Can family planning programs reduce high desired family size in sub-Saharan Africa? Int Perspect Sex Reprod Health 37:209-216. https://doi.org/10.1363/3720911 
Bygbjerg I (2012) Double burden of noncommunicable and infectious diseases in developing countries. Science 337:1499-1501

Castles S (2013) The forces driving global migration. J Intercult Stud 34:122-140. https://doi.org/10.1080 /07256868.2013.781916

Crespo Cuaresma J (2017) Income projections for climate change research: a framework based on human capital dynamics. Glob Environ Chang 42:226-236. https://doi.org/10.1016/j.gloenvcha.2015.02.012

Crespo Cuaresma J, Lutz W (2015) The demography of human development and climate change vulnerability: a projection exercise. Vienna Yearbook Populat Res 13:241-261

Dellink R, Chateau J, Lanzi E, Magné B (2017) Long-term economic growth projections in the shared socioeconomic pathways. Glob Environ Chang 42:200-214. https://doi.org/10.1016/j. gloenvcha.2015.06.004

Ebi KL, Hasegawa T, Hayes K et al (2018) Health risks of warming of $1.5{ }^{\circ} \mathrm{C}, 2{ }^{\circ} \mathrm{C}$, and higher, above preindustrial temperatures. Environ Res Lett 13:063007

EPA (2013) United States Environmental Protection Agency, 78 FR 3085

Foreman KJ, Marquez N, Dolgert A et al (2018) Forecasting life expectancy, years of life lost, and all-cause and cause-specific mortality for 250 causes of death: reference and alternative scenarios for 2016-40 for 195 countries and territories. Lancet 392:2052-2090

Gasparrini A, Guo Y, Hashizume M et al (2015) Mortality risk attributable to high and low ambient temperature: a multicountry observational study. Lancet 386:369-375

Gerland P, Raftery AE, Ševčíková H et al (2014) World population stabilization unlikely this century. Science 346:234. https://doi.org/10.1126/science.1257469

Hales S, Kovats S, Lloyd SJ, Campbell-Lendrum D (2014) Quantitative risk assessment of the effects of climate change on selected causes of death, 2030s and 2050s. WHO Press, Geneva

Hasegawa T, Fujimori S, Takahashi K et al (2016) Economic implications of climate change impacts on human health through undernourishment. Clim Chang 136:189-202. https://doi.org/10.1007/s10584-016-1606-4

Hess JJ, Ebi KL (2016) Iterative management of heat early warning systems in a changing climate. Ann N Y Acad Sci 1382:21-30. https://doi.org/10.1111/nyas.13258

Howard G, Calow R, Macdonald A, Bartram J (2016) Climate change and water and sanitation: likely impacts and emerging trends for action. Annu Rev Environ Resour 41:253-276

Hughes B, Kuhn R, Peterson CM et al (2011) Projections of global health outcomes from 2005 to 2060 using the international futures integrated forecasting model. Bull World Health Organ 89:478-486

Hughes B, Peterson CM, Rothman DS, Solorzano JR (2014) IFs health model documentation. Pardee Center for International Futures, Denver

Hughes BB (2019) International futures: building and using global models. Elsevier Science

IPCC (2000) Special report on emissions scenarios. Cambridge University Press, Cambridge

Jerneck A, Olsson L (2013) A smoke-free kitchen: initiating community based co-production for cleaner cooking and cuts in carbon emissions. J Clean Prod 60:208-215. https://doi.org/10.1016/j.jclepro.2012.09.026

Jiang L, O’Neill BC (2017) Global urbanization projections for the shared socioeconomic pathways. Glob Environ Chang 42:193-199. https://doi.org/10.1016/j.gloenvcha.2015.03.008

KC S, Lutz W (2017) The human core of the shared socioeconomic pathways: population scenarios by age, sex and level of education for all countries to 2100. Glob Environ Chang 42:181-192. https://doi.org/10.1016/j. gloenvcha.2014.06.004

Keim ME (2008) Building human resilience: the role of public health preparedness and response as an adaptation to climate change. Am J Prev Med 35:508-516

Kuhn R, Rothman DS, Turner S et al (2016) Beyond attributable burden: estimating the avoidable burden of disease associated with household air pollution. PLoS One: 11

Landrigan PJ, Fuller R, Acosta NJR et al (2018) The Lancet Commission on pollution and health. Lancet 391: 462-512. https://doi.org/10.1016/S0140-6736(17)32345-0

Lass W, Haas A, Hinkel J, Jaeger C (2011) Avoiding the avoidable: towards a European heat waves risk governance. Int J Disaster Risk Sci 2:1-14

Lloyd SJ, Kovats RS, Chalabi Z (2011) Climate change, crop yields, and undernutrition: development of a model to quantify the impact of climate scenarios on child undernutrition. Environ Health Perspect 119:1817-1823

Lutz W, Butz WP, KC S (eds) (2014a) World population and human capital in the twenty-first century. Oxford University Press, Oxford

Lutz W, KC S (2011) World population and human capital in the twenty-first century. Science 333:587-592. https://doi.org/10.1126/science.1206964

Lutz W, Muttarak R, Striessnig E (2014b) Universal education is key to enhanced climate adaptation. Science 346:1061-1062

Markandya A, Sampedro J, Smith SJ et al (2018) Health co-benefits from air pollution and mitigation costs of the Paris Agreement: a modelling study. Lancet Planetary Health 2:e126-e133 
Marmot M (2005) Social determinants of health inequalities. Lancet 365:1099-1104. https://doi.org/10.1016 /S0140-6736(05)71146-6

Mathers CD, Loncar D (2006) Projections of global mortality and burden of disease from 2002 to 2030. PLoS Med 3:e442

Mathers CD, Loncar D (2005) Updated projections of global mortality and burden of disease, 2002-2030: data sources, methods and results. World Health Organization, Geneva

O’Neill BC, Kriegler E, Ebi KL et al (2017) The roads ahead: narratives for shared socioeconomic pathways describing world futures in the 21st century. Glob Environ Chang 42:169-180. https://doi.org/10.1016/j. gloenvcha.2015.01.004

Orman AR (2005) The epidemiologic transition: a theory of the epidemiology of population change. Milbank Quarter 83:731-757. https://doi.org/10.1111/j.1468-0009.2005.00398.x

Roth GA, Abate D, Abate KH et al (2018) Global, regional, and national age-sex-specific mortality for 282 causes of death in 195 countries and territories, 1980-2017: a systematic analysis for the Global Burden of Disease Study 2017. Lancet 392:1736-1788. https://doi.org/10.1016/S0140-6736(18)32203-7

Sellers S, Ebi KL (2018) Climate change and health under the shared socioeconomic pathway framework. Int J Environ Res Public Health 15:3

Shaffer R, Sellers S, Baker MG et al (2019) Improving and expanding estimates of the global burden of disease due to environmental health risk factors. Environ Health Perspect 127:105001

Stanaway JD, Afshin A, Gakidou E et al (2018) Global, regional, and national comparative risk assessment of 84 behavioural, environmental and occupational, and metabolic risks or clusters of risks for 195 countries and territories, 1990-2017: a systematic analysis for the Global Burden of Disease Study 2017. Lancet 392: 1923-1994. https://doi.org/10.1016/S0140-6736(18)32225-6

United Nations (2019) World population prospects 2019. United Nations, New York

United Nations (2018) World urbanization prospects: the 2018 revision

Van Vuuren DP, Edmonds J, Kainuma M et al (2011) The representative concentration pathways: an overview. Clim Chang 109:5-31

van Vuuren DP, Kriegler E, O’Neill BC et al (2014) A new scenario framework for climate change research: scenario matrix architecture. Clim Chang 122:373-386. https://doi.org/10.1007/s10584-013-0906-1

Watts N, Amann M, Arnell N et al (2019) The 2019 report of the Lancet countdown on health and climate change: ensuring that the health of a child born today is not defined by a changing climate. Lancet 394 : 1836-1878. https://doi.org/10.1016/S0140-6736(19)32596-6

Wheeler T, von Braun J (2013) Climate change impacts on global food security. Science 341:508. https://doi. org/10.1126/science.1239402

World Bank (2018) World development indicators 2018, Washington DC

Publisher's note Springer Nature remains neutral with regard to jurisdictional claims in published maps and institutional affiliations. 